\title{
Pseudo-point-group approach to stereochemistry and stereoisomerism of cyclohexane derivatives
}

\author{
By Shinsaku Fujita \\ Department of Chemistry and Materials Technology, Kyoto Institute of Technology, \\ Goshokaido-cho, Matsugasaki, Sakyo-ku, Kyoto 606-8585
}

(Communicated by Hitosi NozakI, M. J. A., Dec. 12, 2001)

\begin{abstract}
A pseudo-point group $\widehat{\boldsymbol{D}}_{6 h}$ and its subgroups are applied to the symmetry characterization of tri- and tetra-substituted cyclohexane derivatives under flexible and fixed conditions. After the subgroups are classified into chiral and achiral ones as well as into isoenergetic and anisoenergetic ones, the conformational changes of the derivatives are categorized into isoenergetic-achiral (Types I and I'), isoenergetic-chiral (Type II), anisoenergetic-achiral (Type III), and anisoenergetic-chiral cases (Type IV). These cases are rationalized by the subduction from $\widehat{\boldsymbol{D}}_{6 h}$ to $\boldsymbol{D}_{3 d}$.
\end{abstract}

Key words: Cyclohexane; conformer; pseudo-point group; isoenergetic subgroup.

Introduction. Stereochemistry and stereoisomerism of cyclohexane and related compounds have caused some difficulties to organic chemists in comprehending the whole aspects, because of the flexible nature of a cyclohexane skeleton. In order to develop a comprehensive method, we should take account of the following items: (1) the symmetry of cyclohexane itself and the symmetry of every cyclohexane derivative to be examined, (2) whether two chair-form conformers for a cyclohexane derivative are energetically equivalent or not, (3) the chirality/achirality as conformers and as compounds, (4) coherency under considering combinatorial enumeration of isomers, (5) whether two ligands (or substituents) are equivalent or not under fixed conditions and under flexible conditions, and (6) whether two ligands (or substituents) are equivalent or not under reflection operations.

Although various methodologies have been reported, they have succeeded only partially in explaining the items described above. For example, the symmetry of cyclohexane has been discussed conventionally in terms of "averaged symmetry", as ElielWilen's textbook on stereochemistry (Section 4-5) $)^{1)}$ has claimed. Obviously, the conventional way using the $\boldsymbol{D}_{6 h}$-symmetry as an averaged symmetry lacks a tool for specifying stereochemistry in a cyclohexane molecule (Item 5) and a methodology for characterizing stereoisomerism among conformers (Item 2).

Leonard et al..$^{2), 3)}$ have reported an enumeration of cyclohexanes by combining the Pólya-Redfield theorem with extended point groups. An alternative demonstration reported by Flurry ${ }^{4), 5}$ is based on an isodynamic operator (or the corresponding group $\mathbf{F}$ ), which was presented by Altmann. ${ }^{6)}$ Although these two methods have assigned the flipping cyclohexane itself to the group $\boldsymbol{D}_{3 d} R_{6},{ }^{2), 3)}$ or to the group $\mathbf{F} \times \boldsymbol{D}_{3 d}{ }^{4)}$ in place of the conventional $\boldsymbol{D}_{6 h}$, they have not identified the symmetries of cyclohexane derivatives (Item 1).

The chirality/achirality of each cyclohexane derivative (Item 3) has been usually discussed apart from the symmetrical consideration (Item 1). As a typical example of such separate discussion, Morrison-Boyd's textbook ${ }^{7}$ ) has at first depicted a chair-form conformation of cis-1,2-dimethylcyclohexane and then its mirror conformation for comparison. These have been concluded to be conformationally enantiomers, since these are not superimposable but interconvertible. This method requires a distinct discussion on every compound so that tedious efforts must be done to treat more complicated derivatives such as tri- or more-substituted cyclohexanes.

To comprehend the stereochemistry and stereo- 
Table I. Isoenergetic and anisoenergetic subgroups of $\widehat{\boldsymbol{D}}_{6 h}$

\begin{tabular}{ccc}
\hline & Chiral & Achiral \\
\hline Anisoenergetic & Type IV $(\mathrm{Q} \rightleftarrows \mathrm{R}$ or $\overline{\mathrm{Q}} \rightleftarrows \overline{\mathrm{R}})$ & Type III $(\mathrm{A} \rightleftarrows \mathrm{B})$ \\
& $\boldsymbol{C}_{1}, \boldsymbol{C}_{2}^{\prime}, \boldsymbol{C}_{3}, \boldsymbol{D}_{3}$ & $\boldsymbol{C}_{s}, \boldsymbol{C}_{i}, \boldsymbol{C}_{2 h}, \boldsymbol{C}_{3 v}, \boldsymbol{C}_{3 i}, \boldsymbol{D}_{3 d}$ \\
Isoenergetic & Type II $(\mathrm{Q} \rightleftarrows \mathrm{Q}$ or $\overline{\mathrm{Q}} \rightleftarrows \overline{\mathrm{Q}})$ & Type I $(\mathrm{A} \rightleftarrows \mathrm{A})$ \\
& $\widehat{\boldsymbol{C}}_{2}, \widehat{\boldsymbol{C}}_{2}^{\prime}, \widehat{\boldsymbol{D}}_{2}, \widehat{\boldsymbol{C}}_{6}, \widehat{\boldsymbol{D}}_{3}, \widehat{\boldsymbol{D}}_{6}$ & $\widehat{\boldsymbol{C}}_{2 v}, \widehat{\boldsymbol{C}}_{2 v}^{\prime}, \widehat{\boldsymbol{C}}_{2 h}, \widehat{\boldsymbol{C}}_{2 h}^{\prime}, \widehat{\boldsymbol{D}}_{2 h}$, \\
& $\widehat{\boldsymbol{C}}_{6 v}, \widehat{\boldsymbol{C}}_{6 h}, \widehat{\boldsymbol{D}}_{3 h}, \widehat{\boldsymbol{D}}_{3 d}, \widehat{\boldsymbol{D}}_{6 h}$ \\
& Type I' $\left.{ }^{\prime} \rightleftarrows \mathrm{Q}\right)$ \\
& $\widehat{\boldsymbol{C}}_{s}, \widehat{\boldsymbol{C}}_{s}^{\prime}, \widehat{\boldsymbol{C}}_{2 v}^{\prime \prime}, \widehat{\boldsymbol{C}}_{3 v}, \widehat{\boldsymbol{C}}_{3 h}, \widehat{\boldsymbol{D}}_{3 h}^{\prime}$ \\
&
\end{tabular}

isomerism of cyclohexane derivatives, we have defined the pseudo-point group $\widehat{\boldsymbol{D}}_{6 h}$, where sphericity and chronality have been shown to be useful concepts. ${ }^{8), 9)}$ The method based on pseudopoint groups has been successfully applied to trioxane derivatives, ${ }^{8)}$ dioxane derivaitves, ${ }^{10), 11)}$ spiro[5.5]undecane derivatives, ${ }^{12)}$ and piperidine derivatives. ${ }^{13)}$ These previous papers have revealed that the method is useful to discuss stereoismerism and combinatorial enumeration (Items 1 to 4 ) as well as Items 5 and 6 for stereochemistry. However, further investigation is necessary, since we have recently proposed a new standpoint to discuss stereoisomerism and stereochemistry. ${ }^{14)}$ Hence, the present paper will be devoted to discuss the stereoisomerism and stereochemistry of cyclohexane derivatives from the new standpoint, where the importance of three kinds of maximal subgroups will be emphasized.

Formulation of pseudo-point groups. To formulate axial-equatorial exchange in a cyclohexane skeleton, we consider a pair of chair-form conformers. Then, we introduce a pseudo-dihedral rotation $\left(\widehat{C}_{2}^{\prime}-\right.$ operation), which is a combination of flipping and exchange. ${ }^{9)}$ By considering the operation $\widehat{C}_{2}^{\prime}$ and the point group $\boldsymbol{D}_{3 d}$, we can construct the set $\widehat{\boldsymbol{D}}_{6 h}$,

$$
\begin{aligned}
\widehat{\boldsymbol{D}}_{6 h}= & \boldsymbol{D}_{3 d}+\widehat{C}_{2(1)}^{\prime} \boldsymbol{D}_{3 d}, \\
=\{ & I, C_{3}, C_{3}^{2}, S_{6}, i, S_{6}^{5}, C_{2(1)}^{\prime}, C_{2(2)}^{\prime}, C_{2(3)}^{\prime}, \\
& \sigma_{v(1)}, \sigma_{v(2)}, \sigma_{v(3)}, \widehat{C}_{2(1)}^{\prime}, \widehat{C}_{2(2)}^{\prime}, \widehat{C}_{2(3)}^{\prime}, \\
& \widehat{\sigma}_{v(3)}, \widehat{\sigma}_{v(1)}, \widehat{\sigma}_{v(2)}, \widehat{C}_{2}, \widehat{C}_{6}^{5}, \widehat{C}_{6}, \\
& \left.\widehat{\sigma}_{h}, \widehat{S}_{3}, \widehat{S}_{3}^{5}\right\},
\end{aligned}
$$

where we place $\widehat{C}_{2(1)}^{\prime}=\widehat{C}_{2}^{\prime}$ for distinguishing from other operations of the same kind. The resulting set $\widehat{\boldsymbol{D}}_{6 h}$ is called a pseudo-point group. The oper- ators contained in the $\widehat{C}_{2(1)}^{\prime} \boldsymbol{D}_{3 d}$ are called pseudorotations, which are designated by symbols with a hat (circumflex). On the other hand, the operators of $\boldsymbol{D}_{3 d}$ are simply called rotations or more distinctly usual rotations, which are designated by symbols without a hat. As the usual rotations are classified into proper and improper rotations, the pseudorotations are classified into proper and improper rotations. Thereby, the operators of the pseudo-point group $\widehat{\boldsymbol{D}}_{6 h}$ are classified into four classes.

Isoenergetic and anisoenergetic groups. By starting from the 24 operations of $\widehat{\boldsymbol{D}}_{6 h}$, we can construct its subgroups, where a set of conjugate subgroups are enumerated once. They are classified into two categories, i.e., isoenergetic and anisoenergetic subgroups (Table I), by virtue of rotations or pseudo-rotations. Thus, if a subgroup contains at least one pseudo-rotation (proper pseudo-rotation or pseudo-rotoreflection), it is defined as an isoenergetic subgroup; otherwise it is defined as being anisoenergetic. Such an isoenergetic subgroup is designated by a symbol with a hat, while an anisoenergetic subgroup is designated by a symbol without a hat. These subgroups can be alternatively categorized into chiral and achiral subgroups by virtue of proper or improper (pseudo-)rotations. Thereby, we obtain four categories of subgroups, as shown in Table I: chiral-anisoenergetic subgroups (Type IV), achiral-anisoenergetic subgroups (Type III), chiralisoenergetic subgroups (Type II), and achiralisoenergetic subgroups. ${ }^{9)}$

The achiral-isoenergetic subgroups can be further categorized into two cases (Type I and Type I'). If an achiral-isoenergetic subgroup contains no proper pseudo-rotations, it is defined as a Type $\mathrm{I}^{\prime}$ 


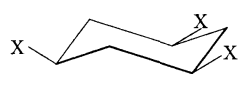

$1 \mathrm{a}$

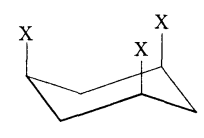

$1 \mathrm{~b}$
Fig. 1. $r-1, c-3, c-5$-Tri-substituted cyclohexane.

subgroup; otherwise, it is defined as a Type I subgroup.

Among the subgroups of $\widehat{\boldsymbol{D}}_{6 h}$ (order 24), three subgroups of order 12 should be mentioned. The first one is $\boldsymbol{D}_{3 d}$, which is called the maximal anisoenergetic subgroup. This group and its subgroup are related to the fixation of a cyclohexane ring. The second one is $\widehat{\boldsymbol{D}}_{6}$, which is called the maximal chiral subgroup. This group and its subgroups are related to the chirality/achirality phenomena. The third one is $\widehat{D}_{3 h}^{\prime}$, which is called the maximal pseudo-reflective subgroup. This group and its subgroups are related to the nature of internal racemization.

Symmetries of tri-substituted cyclohexane derivatives. The combinatorial enumeration by the USCI method shows the presence of total 12 isomers of tri-substituted cyclohexane derivatives $\left(\mathrm{C}_{6} \mathrm{H}_{9} \mathrm{X}_{3}\right)$ : seven $\boldsymbol{C}_{1^{-}}$, four $\boldsymbol{C}_{\boldsymbol{s}^{-}}$, and one $\boldsymbol{C}_{3 v^{-}}$ isomer. ${ }^{9)}$ They are all anisoenergetic, as found by the absence of a hat on each symbol.

The symmetry of $r-1, c-3, c-5$-triX-cyclohexane (1a/1b) is assigned to $C_{3 v}$, which is anisoenergetic and achiral (Type III). According to the general equation $\left(\mathrm{A} \rightleftarrows \mathrm{B}\right.$ ), the conformer 1 a of $\boldsymbol{C}_{3 v}$ point group is converted into the conformer $\mathbf{1 b}$ of $\boldsymbol{C}_{3 v}$ point group, where the two conformers are energetically different from each other (Fig. 1).

The four $\boldsymbol{C}_{s}$-tri-substituted isomers are determined to be $r-1, c-3, c-5-(\mathbf{2 a} / \mathbf{2 b}), r-1, c-2, c-3-$ $(\mathbf{3 a} / \mathbf{3 b}), r-1, t-2, c-3-(\mathbf{4 a} / \mathbf{4 b})$, and 1,1,4-triX-cyclohexane $(\mathbf{5 a} / \mathbf{5 b})$, as shown in Fig. 2. The two achiral conformers of each pair are energetically different. This fact agrees with the general equation $(\mathrm{A} \rightleftarrows \mathrm{B})$ for a Type III case (Table I).

The seven tri-substituted isomers of $\boldsymbol{C}_{1}$-symmetry are determined to be four 1,2,4-, a 1,1,2-, a 1,1,3-, and an $r-1, c-2, t$-3-isomer. These isomers are chiral and anisoenergetic (Type III), as shown in Table I.

Symmetries of tetra-substituted cyclohexane derivatives. The combinatorial enumeration by the USCI method shows that there are totally 32

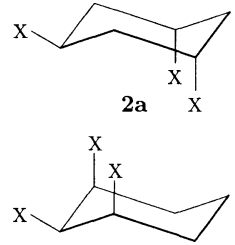

$3 \mathbf{a}$

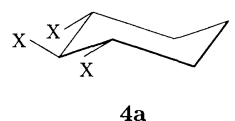

$4 \mathbf{a}$

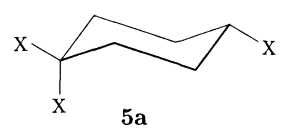

$C_{s}$ (Type III)

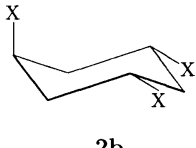

$2 \mathrm{~b}$

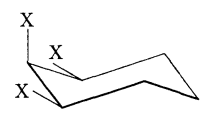

$3 \mathbf{b}$

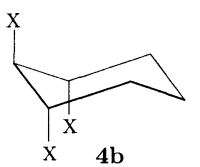

$4 \mathrm{~b}$

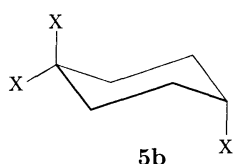

Fig. 2. Tri-substituted cyclohexanes of $\boldsymbol{C}_{\boldsymbol{s}}$-symmetry.
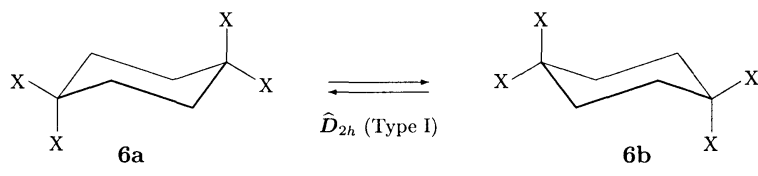

Fig. 3. 1,1,4,4-Tetra-substituted cyclohexane.

isomers of tetra-substituted cyclohexane derivatives $\left(\mathrm{C}_{6} \mathrm{H}_{8} \mathrm{X}_{4}\right): 13 \boldsymbol{C}_{1^{-}}$, two $\widehat{\boldsymbol{C}}_{2^{-}}^{\prime}$, two $\boldsymbol{C}_{2^{-}}^{\prime}$, six $\boldsymbol{C}_{s^{-}}$, two $\widehat{\boldsymbol{C}}_{s^{-}}$, one $\widehat{\boldsymbol{D}}_{2^{-}}$, one $\widehat{\boldsymbol{C}}_{2 v^{-}}$, one $\widehat{\boldsymbol{C}}_{2 v^{-}}^{\prime}$, one $\widehat{\boldsymbol{C}}_{2 v^{-}}^{\prime \prime}$, one $\widehat{\boldsymbol{C}}_{2 h^{-}}^{\prime}$, one $\boldsymbol{C}_{2 h^{-}}$, and one $\widehat{\boldsymbol{D}}_{2 h^{-}}$isomer. ${ }^{9)}$

The $\widehat{\boldsymbol{D}}_{2 h}$-isomer is determined to be $1,1,4,4$ tetraX-cyclohexane $(\mathbf{6 a} / \mathbf{6 b})$, which belongs to Type I, as shown in Fig. 3. According to the general equation ( $\mathrm{A} \rightleftarrows \mathrm{A}$ ), the conformer $\mathbf{6 a}$ of $\boldsymbol{C}_{2 h}$ point group is converted into a homomeric conformer $\mathbf{6 b}$ of $\boldsymbol{C}_{2 h}$ point group. It should be noted that the pseudo-point group $\widehat{D}_{2 h}$ is assigned to the $\mathbf{6 a} / \mathbf{6 b}$ pair, but not to each conformer.

$r-1, t-2, t-4-, c-5$-TetraX-cyclohexane $(\mathbf{7 a} / \mathbf{7 b})$ is assigned to the $\boldsymbol{C}_{2 h}$-symmetry (Fig. 4). According to the general equation $(\mathrm{A} \rightleftarrows \mathrm{B}$ ) for Type III, the conformer $\mathbf{7 a}$ of $\boldsymbol{C}_{2 h}$ point group is converted into the conformer $\mathbf{7 b}$ of $\boldsymbol{C}_{2 h}$ point group, where the two conformers are energetically different from each other.

The $\widehat{\boldsymbol{C}}_{2 h}^{\prime}$-isomer is decided to be $r-1, c-2, t-4-$, $t$-5-tetraX-cyclohexane $(\mathbf{8} \mathbf{a} / \mathbf{8} \mathbf{b})$, which belongs to Type I as shown in Fig. 4. According to the general equation $(\mathrm{A} \rightleftarrows \mathrm{A}$ ) for Type $\mathrm{I}$, the conformer $\mathbf{8 a}$ of $C_{s}$ point group is converted into the homomeric conformer $\mathbf{8 b}$ of $C_{s}$ point group, while the $\mathbf{8 a} / \mathbf{8 b}$ 

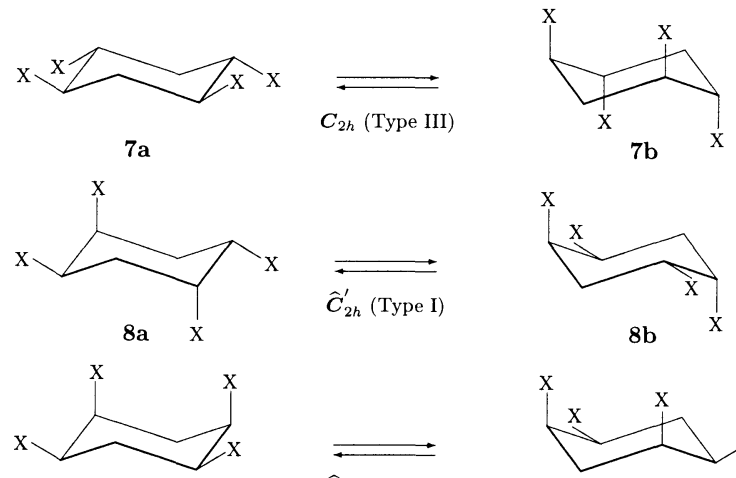

9a
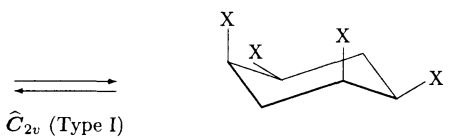

$9 b$

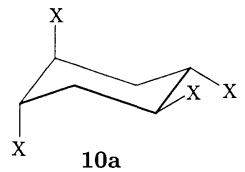

$\stackrel{\rightleftarrows}{\widehat{D}_{2} \text { (Type II) }}$

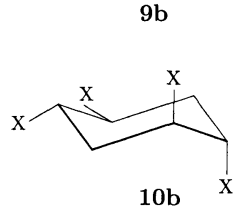

Fig. 4. 1,2,4,5-TetraX-cyclohexanes.
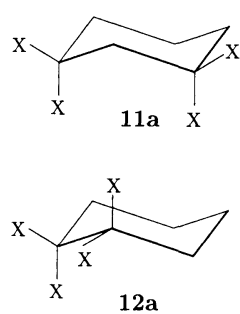
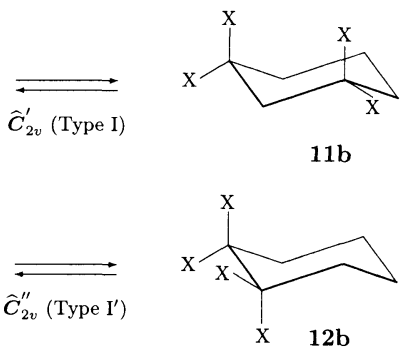

Fig. 5. 1,1,3,3- and 1,1,2,2-Tetra-substituted cyclohexanes.

pair belongs to the pseudo-point group $\widehat{C}_{2 h}^{\prime}$.

The $\widehat{\boldsymbol{C}}_{2 v}$-isomer is determined to be $r-1, c-2$, $c$-4-,c-5-tetraX-cyclohexane $(\mathbf{9 a} / \mathbf{9} \mathbf{b})$, which belongs to Type I, as shown in Fig. 4. The same symmetrical discussion as the case of the $\mathbf{8 a} / \mathbf{8 b}$ case is available for this case.

The symmetry of $r-1, t-2, c-4-, t-5$-tetraX-cyclohexane $(\mathbf{1 0 a} / \mathbf{1 0 b})$ is found out to be $\widehat{D}_{2}$, which is categorized to a Type II case (Table I). Since this pair is in agreement with the general equation $(\mathrm{Q} \rightleftarrows \mathrm{Q})$, there appears the enantiomeric pair corresponding to the equation $(\overline{\mathrm{Q}} \rightleftarrows \overline{\mathrm{Q}})$.

The $\widehat{\boldsymbol{C}}_{2 v}^{\prime}$-symmetry (Type I) is assigned to 1,1,3,3-tetraX-cyclohexane $(\mathbf{1 1} \mathbf{a} / \mathbf{1 1} \mathbf{b})$, as illustrated in Fig. 5. In accord with the general equation $(\mathrm{A} \rightleftarrows$ A) for Type I, the conformer 11a of $C_{s}$ point group is converted into a homomeric conformer $11 \mathrm{~b}$ of $C_{s}$ point group.

1,1,2,2-TetraX-cyclohexane (12a/12b) belongs
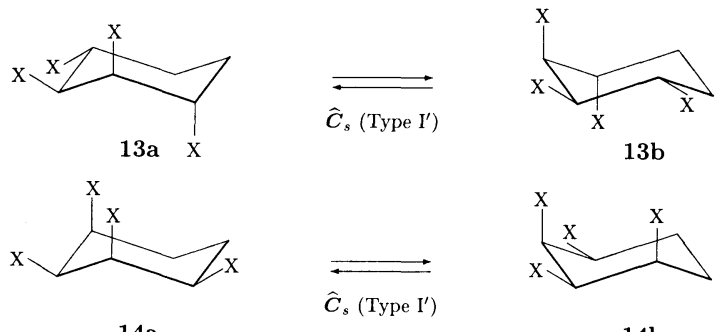

$14 \mathrm{~b}$

Fig. 6. 1,2,3,4-Tetra-substituted cyclohexanes of $\widehat{\boldsymbol{C}}_{\boldsymbol{s}}$-symmetry.
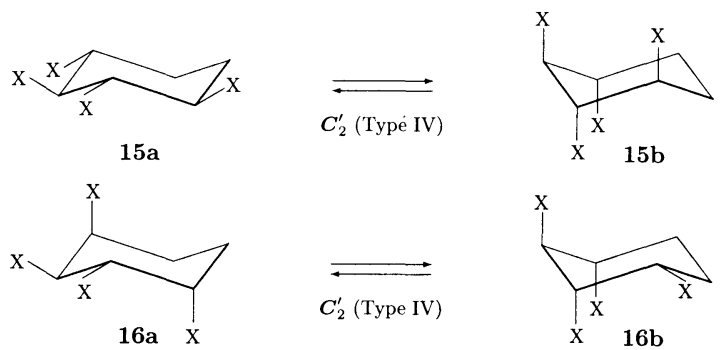

Fig. 7. 1,2,3,4-Tetra-substituted cyclohexanes of $\boldsymbol{C}_{2}^{\prime}$-symmetry.

to the $\widehat{\boldsymbol{C}}_{2 v}^{\prime \prime}$-symmetry (Type $\mathrm{I}^{\prime}$ ), as depicted in Fig. 5. The conformer 12a belonging to the chiral point group $C_{2}$ is enantiomeric to the conformer of the opposite chirality $(\mathbf{1 2 b})$ in agreement with the general expression $\mathrm{Q} \rightleftarrows \overline{\mathrm{Q}}$ for Type $\mathrm{I}^{\prime}$.

The two isomers of $\widehat{C}_{s}$-symmetry are determined to be $r-1, t-2, t-3-, c-4-(\mathbf{1 3 a} / \mathbf{1 3} \mathbf{b})$ and $r-1, c-$ 2,c-3-,c-4-tetraX-cyclohexane (14a/14b), as shown in Fig. 6. These isomers are in agreement with the general expression $\mathrm{Q} \rightleftarrows \overline{\mathrm{Q}}$ for Type $\mathrm{I}^{\prime}$. Although each of the conformers (under fixed conditions) belongs to the point group $C_{1}$, the pseudo-point group $\widehat{C}_{s}$ indicates the achirality under flexible conditions.

The two isomers of $C_{2}^{\prime}$-symmetry are found out to be $r-1, t-2, c-3-, t-4-(\mathbf{1 5 a} / \mathbf{1 5} \mathbf{b})$ and $r-1, c-2, t-3-$, $t$-4-tetraX-cyclohexane $(\mathbf{1 6 a} / \mathbf{1 6} \mathbf{b})$, as shown in Fig. 7. These isomers satisfy the general expression $\mathrm{Q} \rightleftarrows \mathrm{R}$ for Type IV. Each of the conformers (under fixed conditions) belongs to the point group $C_{2}^{\prime}$, which is isomorphic to the pseudo-point group $\boldsymbol{C}_{2}^{\prime}$ for flexible conditions.

The two isomers of $\widehat{\boldsymbol{C}}_{2}^{\prime}$-symmetry are decided to be $r-1,2,2-, t$-3-tetraX-cyclohexane $(\mathbf{1 7 a} / \mathbf{1 7} \mathbf{b})$ and 1,1-r-3,t-5-tetraX-cyclohexane $(\mathbf{1 8 a} / \mathbf{1 8} \mathbf{b})$, as illustrated in Fig. 8. They are categorized to Type II cases (Table I). Since each of the pairs is in agreement 

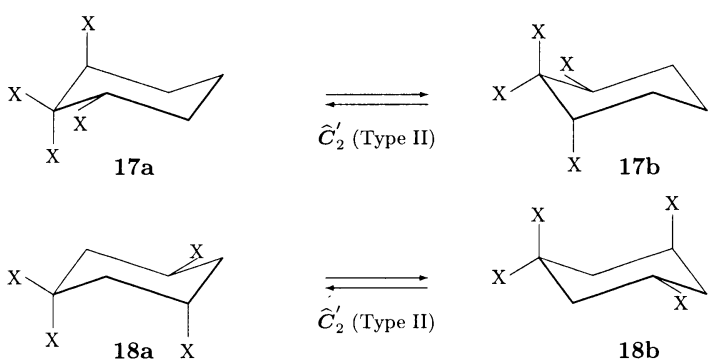

$\stackrel{\longrightarrow}{\hat{C}_{2}^{\prime} \text { (Type II) }}$

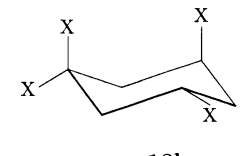

$18 \mathrm{~b}$

Fig. 8. Tetra-substituted cyclohexanes of $\widehat{\boldsymbol{C}}_{2}^{\prime}$-symmetry.
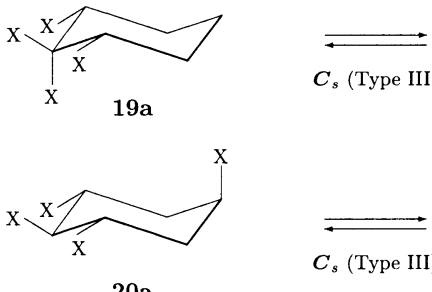

$C_{s}$ (Type III

20
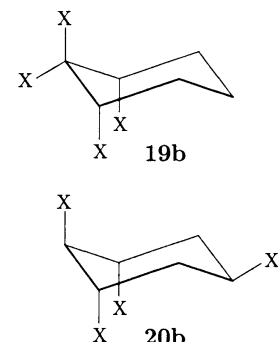

$\mathrm{X} \quad 20 \mathrm{~b}$
Fig. 9. Examples of tetra-substituted cyclohexanes of $\boldsymbol{C}_{s}$-symmetry.

with the general equation $(\mathrm{Q} \rightleftarrows \mathrm{Q})$, there appears the enantiomeric pair corresponding to the equation $(\overline{\mathrm{Q}} \rightleftarrows \overline{\mathrm{Q}})$.

The six tetra-substituted isomers of $C_{s}$-symmetry are determined to be $r-1,2,2-, c-3$-isomer $(\mathbf{1 9 a} / \mathbf{1 9 b}), \quad 1,1-r-3, c$-5-isomer, $\quad r-1, t-2, c-3-, t-5$-isomer $(\mathbf{2 0 a} / \mathbf{2 0 b}), \quad r-1, c-2, c-3-, c-5$-isomer, $\quad r-1, c-2$, $c$-3-,t-5-isomer, and $r-1, t-2, c-3-, c-5$-isomer, which are all Type III cases. These cases are illustrated with representative examples in Fig. 9.

The 13 tetra-substituted isomers of $\boldsymbol{C}_{1}$-symmetry are determined to be all Type IV cases, though they are not depicted.

Ring fixation. By means of the standpoint introduced by us, ${ }^{14)}$ each cyclohexane derivative of symmetry $\boldsymbol{G}$ (more precisely a set of conformers equivalent under $\left.\widehat{\boldsymbol{D}}_{6 h}\right)$ is represented by a coset representation $\widehat{\boldsymbol{D}}_{6 h}(/ \boldsymbol{G})$, the local symmetry of which is identical with the $\boldsymbol{G}$. Fixation processes of such cyclohexane derivatives are characterized by desymmetrization of $\widehat{\boldsymbol{D}}_{6 h}$ into the maximal anisoenergetic subgroup $\boldsymbol{D}_{3 d}$. They are rationally explaind by the subduction of coset representations (i.e. $\widehat{\boldsymbol{D}}_{6 h}(/ \boldsymbol{G}) \downarrow$ $\boldsymbol{D}_{3 d}$ ) proposed by us. ${ }^{15)-17)}$

Anisoenergetic cases have been characterized by two anisoenergetic conformers ( $Q$ and $\mathrm{R}$ for Type IV; or A and B for Type III), as shown in Table I.

Type IV corresponds to chiral-anisoenergetic subgroups, which are chiral subgroups of $\widehat{\boldsymbol{D}}_{3 h}^{\prime}$ or of $\boldsymbol{D}_{3}$ more restrictively. Hence, two conformers should be chiral. When $\mathrm{Q}$ and $\mathrm{R}$ are such chiral conformers, we have a conformational exchange for Type IV, Q $\rightleftarrows \mathrm{R}$. There exists an enantiomeric process, $\overline{\mathrm{Q}} \rightleftarrows$ $\overline{\mathrm{R}}$, where $\overline{\mathrm{Q}}$ and $\overline{\mathrm{R}}$ are enantiomeric to $\mathrm{Q}$ and $\mathrm{R}$, respectively.

The conclusion can be rationalized by the subduction from $\widehat{\boldsymbol{D}}_{6 h}$ to $\boldsymbol{D}_{3 d}$. The derivative $\mathbf{1 5 a} / \mathbf{1 5 b}$ of Type IV is assigned to a coset representation, $\widehat{\boldsymbol{D}}_{6 h}\left(/ \boldsymbol{C}_{2}^{\prime}\right)$, where the local symmetry $\boldsymbol{C}_{2}^{\prime}$ corresponds to the symmetry of the derivative. The fixation process is represented by

$$
\widehat{\boldsymbol{D}}_{6 h}\left(/ \boldsymbol{C}_{2}^{\prime}\right) \downarrow \boldsymbol{D}_{3 d}=2 \boldsymbol{D}_{3 d}\left(/ \boldsymbol{C}_{2}^{\prime}\right) .
$$

The coefficient (2) and the local symmetry $\left(\boldsymbol{C}_{2}^{\prime}\right)$ on the right-hand side indicate that the fixation process produces two $\boldsymbol{C}_{2}^{\prime}$-conformers ( $\mathrm{Q}$ and $\mathrm{R}$ in general). This is exemplified in Fig. 7.

Type III (achiral-anisoenergetic subgroups) means that two conformers are achiral even under fixed conditions. Hence it corresponds to a process, $\mathrm{A} \rightleftarrows \mathrm{B}$, where $\mathrm{A}$ and $\mathrm{B}$ are achiral conformers.

This conclusion is further explained by the subduction from $\widehat{\boldsymbol{D}}_{6 h}$ to $\boldsymbol{D}_{3 d}$. The derivative $\mathbf{7 a} / \mathbf{7 b}$ of Type III is assigned to a coset representation, $\widehat{\boldsymbol{D}}_{6 h}\left(/ \boldsymbol{C}_{2 h}\right)$, where the local symmetry $\boldsymbol{C}_{2 h}$ corresponds to the symmetry of the derivative. The fixation process is represented by

$$
\widehat{\boldsymbol{D}}_{6 h}\left(/ \boldsymbol{C}_{2 h}\right) \downarrow \boldsymbol{D}_{3 d}=2 \boldsymbol{D}_{3 d}\left(/ \boldsymbol{C}_{2 h}\right),
$$

where the right-hand side indicates that the fixation process produces two $\boldsymbol{C}_{2 h}$-conformers (A and B in general). This is exemplified by the first example depicted in Fig. 4.

The local symmetry in eq. [3] or [4] is invariant during the fixation process. This holds true in anisoenergetic cases, as expressed by the following equation:

$$
\widehat{\boldsymbol{D}}_{6 h}(/ \boldsymbol{G}) \downarrow \boldsymbol{D}_{3 d}=2 \boldsymbol{D}_{3 d}(/ \boldsymbol{G}),
$$

where $G$ is an anisoenergetic subgroup.

Isoenergetic cases have been characterized by two isoenergetic conformers (Q for Type II; A for Type I; or Q and $\overline{\mathrm{Q}}$ for Type $\mathrm{I}^{\prime}$ ), as shown in Table I.

Type II (chiral-isoenergetic subgroups) corresponds to a process, $\mathrm{Q} \rightleftarrows \mathrm{Q}$. There exists an enantiomeric process, $\overline{\mathrm{Q}} \rightleftarrows \overline{\mathrm{Q}}$. 
The fixation process of the derivative $\mathbf{1 0 a} / \mathbf{1 0 b}$ of Type II (Fig. 4) is represented by

$$
\widehat{\boldsymbol{D}}_{6 h}\left(/ \widehat{\boldsymbol{D}}_{2}\right) \downarrow \boldsymbol{D}_{3 d}=\boldsymbol{D}_{3 d}\left(/ \boldsymbol{C}_{2}^{\prime}\right),
$$

where the local symmetry $\widehat{\boldsymbol{D}}_{2}$ on the left-hand side corresponds to the symmetry of the derivative and the local symmetry $\boldsymbol{C}_{2}^{\prime}$ on the right-hand side corresponds to the symmetry of each of the fixed conformers. This is in accord with the general equation $\mathrm{Q} \rightleftarrows \mathrm{Q}$ for Type II shown in Table I.

Type I corresponds to achiral-isoenergetic subgroups that are not contained in $\widehat{\boldsymbol{D}}_{3 h}^{\prime}$. This means a process represented by $\mathrm{A} \rightleftarrows \mathrm{A}$.

The fixation process of the derivative $\mathbf{9 a} / \mathbf{9 b}$ of Type I (Fig. 4) is represented by

$$
\widehat{\boldsymbol{D}}_{6 h}\left(/ \widehat{\boldsymbol{C}}_{2 v}\right) \downarrow \boldsymbol{D}_{3 d}=\boldsymbol{D}_{3 d}\left(/ \boldsymbol{C}_{s}\right),
$$

where the change of the local symmetry $\widehat{\boldsymbol{C}}_{2 v}$ into $\boldsymbol{C}_{\boldsymbol{s}}$ corresponds to the general equation $\mathrm{A} \rightleftarrows \mathrm{A}$ for Type I shown in Table I.

Type I' corresponds to achiral-isoenergetic subgroups that are contained in the maximal pseudorotoreflective subgroup $\widehat{D}_{3 h}^{\prime}$. This means a racemizing process represented by $\mathrm{Q} \rightleftarrows \overline{\mathrm{Q}}$.

The fixation process of the derivative $\mathbf{1 2 a} / \mathbf{1 2 b}$ of Type $\mathrm{I}^{\prime}$ (Fig. 5) is represented by

$$
\widehat{\boldsymbol{D}}_{6 h}\left(/ \widehat{\boldsymbol{C}}_{2 v}^{\prime \prime}\right) \downarrow \boldsymbol{D}_{3 d}=\boldsymbol{D}_{3 d}\left(/ \boldsymbol{C}_{2}^{\prime}\right) .
$$

This means that a conformer pair corresponding to the local symmetry $\widehat{\boldsymbol{C}}_{2 v}^{\prime \prime}$ is converted into conformers of $C_{2}^{\prime}$. This process corresponds to the general equation $\mathrm{Q} \rightleftarrows \overline{\mathrm{Q}}$ for Type $\mathrm{I}^{\prime}$ shown in Table $\mathrm{I}$.

The local symmetry in eqs. [6] to [8] is reduced into a subgroup during the fixation process, where no division of the orbit occurs. Since this is an inherent nature of isoenergetic cases, it holds true in any isoenergetic cases, as expressed by the following equation:

$$
\widehat{D}_{6 h}(/ \boldsymbol{G}) \downarrow \boldsymbol{D}_{3 d}=\boldsymbol{D}_{3 d}\left(/ \boldsymbol{G}^{\prime}\right),
$$

where $G$ is an isoenergetic subgroup and $G^{\prime}$ is the maximal anisoenergetic subgroup of $\boldsymbol{G}$.

Conclusion. The symmetries of cyclohexane derivatives are characterized by a pseudo-point group $\widehat{\boldsymbol{D}}_{6 h}$ and its subgroups, which are classified into chiral and achiral ones as well as into isoenergetic and anisoenergetic ones. Thereby, the conformational changes of the cyclohexane derivatives are categorized into five cases (Table I). These cases are rationalized by the subductions from the global symmetry $\widehat{\boldsymbol{D}}_{6 h}$ to the maximal anisoenergetic subgroup $\boldsymbol{D}_{3 d}$. In particular, the local symmetries contained in the subductions give an insight to comprehend the categorization.

\section{References}

1) Eliel, E. L., and Wilen, S. H. (1994) Stereochemistry of Organic Compounds, John Wiley \& Sons, New York.

2) Leonard, J. E., Hammond, G. S., and Simmons, H. E. (1975) J. Am. Chem. Soc. 97, 5052-5054.

3) Leonard, J. E. (1977) J. Phys. Chem. 81, 2212-2214.

4) Flurry, J. R. L. (1976) J. Phys. Chem. 80, 777-778.

5) Flurry, J. R. L. (1984) J. Chem. Educ. 61, 663-665.

6) Altmann, S. L. (1967) Proc. R. Soc. London, Ser. A 298, 184-203.

7) Morrison, R. T., and Boyd, R. N. (1987) Organic Chemistry. 5th ed., Allyn and Bacon, Boston.

8) Fujita, S. (1994) Bull. Chem. Soc. Jpn. 67, 2927-2934.

9) Fujita, S. (1994) Bull. Chem. Soc. Jpn. 67, 2935-2948.

10) Fujita, S. (1998) J. Chem. Soc. Faraday Trans. 94, 25152523.

11) Fujita, S. (1998) J. Chem. Inf. Comput. Sci. 38, 876-884.

12) Fujita, S. (1998) J. Chem. Soc. Faraday Trans. 94, 31973205.

13) Fujita, S. (1999) Bull. Chem. Soc. Jpn. 72, 1759-1768.

14) Fujita, S. (2001) Bull. Chem. Soc. Jpn. 74, 1585-1603.

15) Fujita, S. (1991) Symmetry and Combinatorial Enumeration in Chemistry, Springer-Verlag, BerlinHeidelberg.

16) Fujita, S. (1989) Theor. Chim. Acta 76, 247-268.

17) Fujita, S. (1990) J. Am. Chem. Soc. 112, 3390-3397. 